\title{
Bilateral pleural effusion associated with atezolizumab in a patient with Lynch syndrome-related urothelial carcinoma: a case report
}

\author{
Ho-Young Yhim ${ }^{1,2} \wedge$, So-Yeon Jeon ${ }^{1,2} \wedge$, Chang-Hoon Lee ${ }^{1,2} \wedge$, Na-Ri Lee ${ }^{1,2} \wedge$ \\ ${ }^{1}$ Division of Hematology and Oncology, Department of Internal Medicine, Jeonbuk National University Hospital-Jeonbuk National University \\ Medical School, Jeonju, Republic of Korea; ${ }^{2}$ Research Institute of Clinical Medicine of Jeonbuk National University-Biomedical Research Institute \\ of Jeonbuk National University Hospital, Jeonju, Republic of Korea \\ Correspondence to: Na-Ri Lee, MD, PhD. Division of Hematology and Oncology, Department of Internal Medicine, Jeonbuk National University \\ Medical School, 634-18, Keumam Dong, Dukjin Gu, Jeonju, Jeonbuk, 561-712, Republic of Korea. Email: nariflower@jbnu.ac.kr.
}

\begin{abstract}
Lynch syndrome (LS) is an inherited syndrome associated with an increased risk of cancer caused by abnormalities in DNA mismatch repair (MMR) genes. Immune checkpoint inhibitors (ICIs) have been reported to lead to a good response in cancers accompanied by LS. However, ICI therapy can cause immune-related adverse events (irAEs). In addition, post ICI treatment, some patients can show a falsely aggravated response, called pseudoprogression, causing difficulties in initial drug response evaluation. A 61-year-old man presented with back and pelvic bone pain. He had a history of surgery for stomach and colon cancer, and his daughter was treated for endometrial cancer. The patient was diagnosed with primary urothelial carcinoma (UC) in the left ureter with adrenal gland and multiple bone metastases. Through nextgeneration sequencing (NGS), mutations in MLH1 and MSH2 were identified, and diagnosis of LS was confirmed. On the $11^{\text {th }}$ day from the start of atezolizumab, left pleural effusion occurred with exacerbation of the rib metastasis; the amount of effusion increased, and percutaneous catheter drainage (PCD) was performed. On the $27^{\text {th }}$ day, right pleural effusion developed, and drainage was initiated. After the third cycle of atezolizumab, the bilateral pleural fluid decreased, and the drainage tube was removed. Positron emission tomography/computed tomography (PET-CT) revealed improvement in the cancer lesions, including metastatic bone lesions. This is a rare case of bilateral pleural effusion due to pseudoprogression of rib lesions after atezolizumab treatment in a patient with ureter cancer accompanied by LS. UC associated with LS is expected to show a good response to ICI therapy. For proper identification of pseudoprogression, appropriate response evaluation and close monitoring of the side effects are necessary.
\end{abstract}

Keywords: Pleural effusion; atezolizumab; Lynch syndrome (LS); urothelial carcinoma (UC); case report

Submitted May 06, 2021. Accepted for publication Jul 13, 2021.

doi: 10.21037/apm-21-1148

View this article at: https://dx.doi.org/10.21037/apm-21-1148

\section{Introduction}

Lynch syndrome (LS) is an autosomal dominant hereditary cancer syndrome that increases the risk of many types of cancer, including colorectal cancer and cancers of the endometrium, stomach, ovary, small bowel, and urinary tract (1). LS can be diagnosed by confirming whether it is accompanied by a germline pathologic variant of mismatch repair (MMR) genes, including $M L H 1, M L H 2, M S H 6$, and PMS2 (1). Defects in MMR proteins result in high microsatellite instability (MSI-H) due to their failure to

^ ORCID: Na-Ri Lee, 0000-0002-6236-9319; Ho-Young Yhim, 0000-0002-1252-5336; So-Yeon Jeon, 0000-0002-4792-5049; Chang-Hoon Lee, 0000-0002-1317-4646. 
repair errors that normally occur during DNA replication (1). MSI-H is known as a predictive factor of the response to immune checkpoint inhibitors (ICIs), and some studies have shown that ICIs show a good response in cancer patients with LS $(2,3)$. Atezolizumab is a monoclonal antibody that interferes with the interaction of programmed death-ligand 1 (PD-L1), programmed death-1 (PD-1) and B7 (4). It was approved by the US Food and Drug Administration as a second-line therapy for advanced urothelial carcinoma (UC).

Interestingly, some patients may show an atypical response to ICI therapy, called pseudoprogression (5). Pseudoprogression is a phenomenon in which the size of the tumor temporarily increases after initiation of ICI therapy and then decreases again with continuation of treatment. The immune response is known to be involved.

We present a rare case of transient bilateral pleural effusion with pseudoprogression of multiple rib metastases that developed after treatment of UC from the left ureter with atezolizumab in LS. We present the following case in accordance with the CARE reporting checklist (available at https://apm.amegroups.com/article/view/10.21037/apm$21-1148 / \mathrm{rc})$.

\section{Case presentation}

A 61-year-old man visited the emergency room for back and pelvic pain a week prior to presentation. Nine years ago, he was diagnosed with early gastric and ascending colon cancer simultaneously. He underwent laparoscopy-assisted distal gastrectomy, Billroth-1, and hemicolectomy, and stages of each cancer were $\mathrm{T} 1 \mathrm{aN} 0$ and $\mathrm{T} 1 \mathrm{~N} 0$, respectively. His family history included a diagnosis of diffuse large B-cell lymphoma in his son and endometrial cancer in his daughter. He was a non-smoker with no history of tuberculosis or autoimmune disease.

Abdominal computed tomography (CT) at admission revealed a $2.8-\mathrm{cm}$ tumor in the left ureter. ${ }^{18}$ Fluoro-2deoxy-D-glucose (FDG) positron emission tomography/ CT $\left({ }^{18}\right.$ F-FDG PET-CT) revealed FDG uptake in the left ureter and adrenal gland, and multiple bone metastases in the skull, ribs, spine, and pelvic bones. A core needle biopsy of the spine revealed UC. Immunohistochemical (IHC) staining for GATA3, CK7, and CK20 was positive.

PD-L1 IHC (SP142) showed an overall low positivity rate ( $<5 \%$ of tumor-infiltrating immune cells). MLH1, MSH2, MSH6, and PMS2 were intact on IHC staining. Next-generation sequencing (NGS) of the hereditary cancer syndrome panel was performed, and MLH1 c.199G>A
(p.Gly67Arg) was identified as a pathological variant. $\mathrm{MSH} 2$ c.2064G>A (p.Met6881le) was detected, and was a variant of uncertain significance. Consequently, the diagnosis of LS was confirmed in the patient.

The patient was treated with cisplatin $\left(70 \mathrm{mg} / \mathrm{m}^{2}\right.$ on day 1$)$ and gemcitabine $\left(1,250 \mathrm{mg} / \mathrm{m}^{2}\right.$ on days 1 and 8$)$ and received palliative radiation therapy from the lumbar spine (L3) to the sacrum. After two cycles, ${ }^{18}$ F-FDG PET-CT revealed progressive disease of the bone lesions (Figure 1).

Second-line immunotherapy (1,200 $\mathrm{mg}$ atezolizumab) was administered at 3-week intervals. The patient's neck and upper back pain worsened, and he required additional radiation therapy from the cervical spine (C7) to the thoracic spine (T1).

Eleven days after atezolizumab administration, the patient was suspected to have pleural effusion due to the general haziness of the left lung observed on a chest radiograph (Figure 2). High-resolution computed tomography (HRCT) revealed moderate to large amounts of left pleural effusion and an increased size of the left rib mass (Figure 3).

The patient's vital signs were as follows: blood pressure, 130/88 $\mathrm{mmHg}$; heart rate, 99 beats/min; respiratory rate, 20 breaths/min; and temperature, $37.0{ }^{\circ} \mathrm{C}$. Transthoracic two-dimensional echocardiography revealed a normal left ventricular ejection fraction of $64 \%$ and no pericardial effusion. The thyroid function test results were normal.

The patient was bedridden and had no symptoms of respiratory distress. The patient was kept under continuous observation as there were no other symptoms; however, as the pleural effusion did not improve, percutaneous catheter drainage (PCD) of the left chest was performed after 9 days. Pleural fluid analysis revealed the following: protein, $2.9 \mathrm{~g} / \mathrm{dL}$ (total serum protein: $5.6 \mathrm{~g} / \mathrm{dL}$ ), lactate dehydrogenase (LDH), 487 IU/L (serum LDH, 1,503 IU/L; normal, 218-472 IU/L); white blood cell count, 141/ $\mathrm{mm}^{3}$ (83\% lymphocytes); and adenosine deaminase (ADA), $15.3 \mathrm{U} / \mathrm{L}$ (normal: 0-40 U/L). His pleural effusion was exudative and lymphocyte-dominant. The infectious workup, including bacterial and viral etiologies, was negative. Cytological examination of the pleural fluid revealed only some inflammatory cells and no malignant cells. Serologic examination for autoimmune diseases (antinuclear antibodies, anti-mitochondrial and smooth muscle antibodies, anti-liverkidney microsomal antibody, and rheumatoid factor) was negative. Approximately 1,000 mL of fluid was drained in 3 days, with 300-400 $\mathrm{mL}$ drained per day (Figure 4).

Twenty-seven days after the first cycle of atezolizumab (6 days after the second cycle), chest $\mathrm{X}$-rays revealed 
A

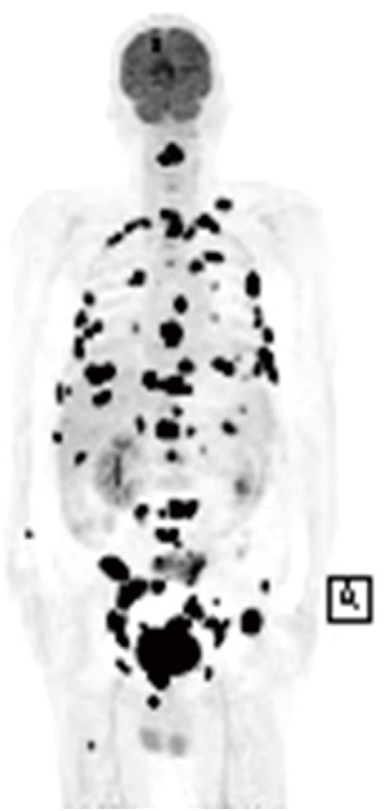

B

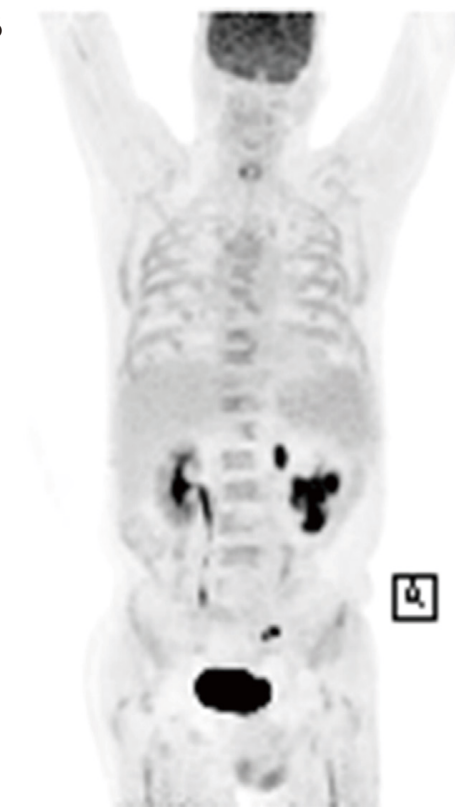

Figure 1 PET-CT findings before and after atezolizumab treatment. (A) PET-CT revealed masses with FDG uptake in the left distal ureter, left adrenal gland, lymph nodes in the left iliac area, and multiple bone metastases in the skull, ribs, spine, left scapula, manubrium, both pelvic bones, and right femur. (B) PET-CT revealed decreased size and metabolism of the ureteric, adrenal gland, lymph node, and multiple bone metastases after the third cycle of atezolizumab. PET-CT, positron emission tomography-computed tomography; FDG, fluoro-2deoxy-D-glucose.

haziness across the right lung (Figure 2), and HRCT revealed a large right pleural effusion, and an increased size of the right and left rib masses was observed (Figure 3). On the following day, right chest PCD was performed, and the amount of pleural fluid drainage was similar to that on the left (Figure 4). Right pleural effusion was also exudative, lymphocyte-dominant, and cytology revealed only some inflammatory cells and mesothelial cells.

The pleural effusion, which continued to drain $300-400 \mathrm{~mL}$ on both sides, suddenly decreased to $50-100 \mathrm{~mL}$, following which the PCD was removed as there was little fluid observed on chest ultrasound 42 days after atezolizumab treatment (Figure 4). The patient's bone pain began to decrease. One week after the third cycle of atezolizumab, an improvement in the bone lesions was observed on PET-CT (Figure 1). The left pleural effusion developed, and $490 \mathrm{~mL}$ was drained after thoracentesis.

Subsequently, pleural effusion was no longer observed on chest radiography (Figure 2).

The patient was able to sit as his neck and back pain was relieved. A fourth cycle of atezolizumab was recently performed; with no suspected pleural effusion.
All procedures performed in this study were in accordance with the ethical standards of the institutional and/or national research committee(s) and with the Helsinki Declaration (as revised in 2013). Written informed consent was obtained from the patient for publication of this case report and accompanying images. A copy of the written consent is available for review by the editorial office of this journal.

\section{Comment}

ICIs have shown marked efficacy in various advanced cancers and improved patient survival.

It should be noted that ICI therapy can cause immunerelated adverse events (irAEs) in all organs due to its immunotherapeutic mechanism. Atezolizumab is associated with various adverse events, including fatigue, nausea, decreased appetite, pruritus, pyrexia, diarrhea, and rash $(4,6)$. In the IMvigor 210 trial, irAEs occurred in $7 \%$ of patients, with pneumonitis, increased liver enzymes, rash, and dyspnea being the most common effects (7). The presence of infiltrating $\mathrm{T}$ lymphocytes in organs affected 

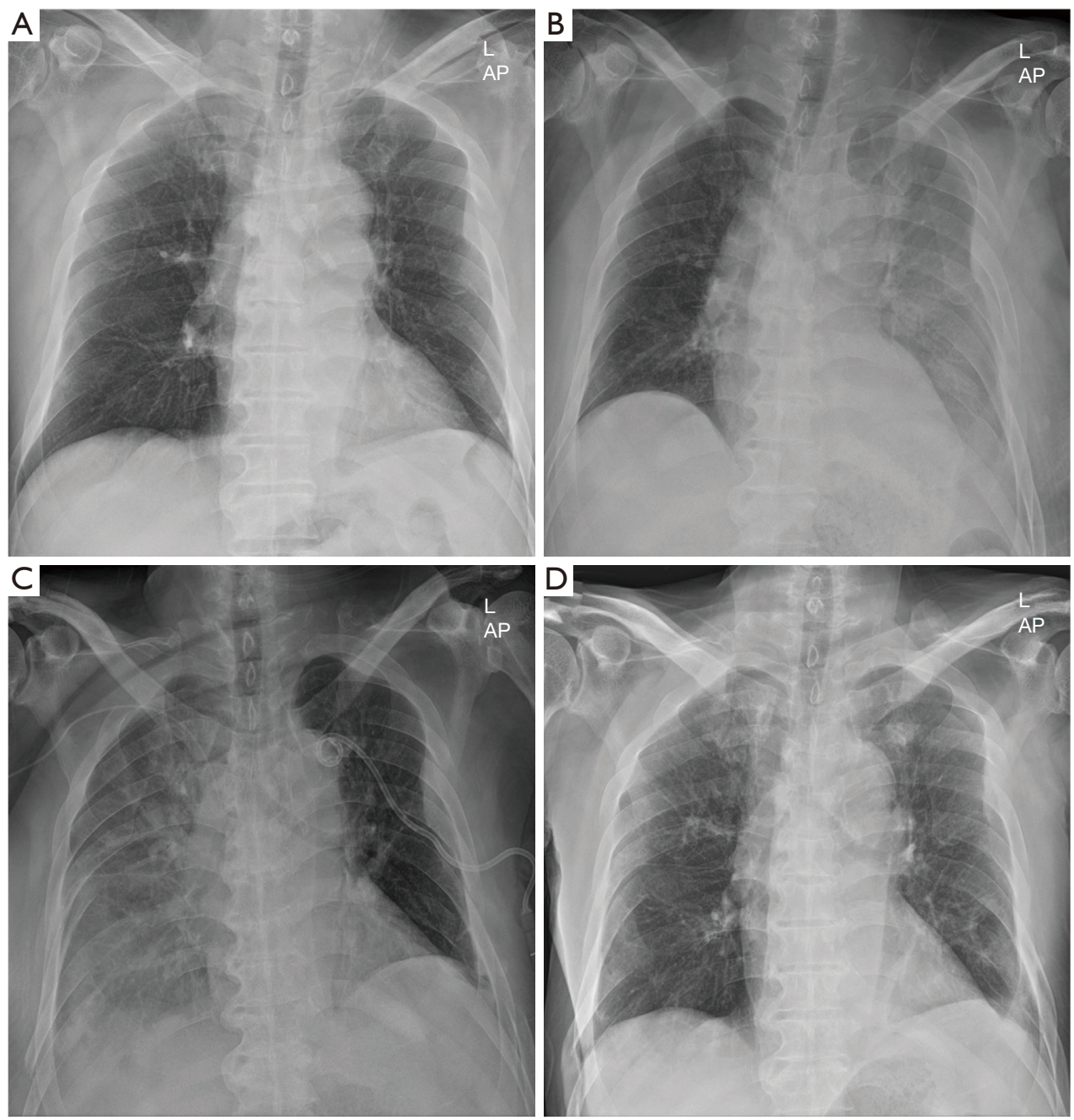

Figure 2 Chest X-ray findings before and after atezolizumab treatment. (A) Chest X-ray showing no pleural effusion 10 days before atezolizumab. (B) Chest X-ray showing diffuse haziness on the left lung field 11 days after atezolizumab. (C) Chest X-ray showing diffuse haziness on the right lung field and percutaneous catheter drainage of the left chest inserted 27 days after the first cycle of atezolizumab (6 days after the second cycle). (D) Chest X-ray showing no diffuse haziness on both lung fields 60 days after the first cycle of atezolizumab (18 days after the third cycle).

by such irAEs suggests that these events are due to cellular immunity mediated by $\mathrm{T}$ cells (4).

A new response pattern to ICIs has been observed, known as pseudoprogression: response to treatment seen after the initial increase in cancer lesion volume due to the infiltration of tumoral tissue by immune cells. Overall, the rate of pseudoprogression did not exceed $10 \%$ in patients treated with ICIs (8).

We considered the possibility of irAEs and pseudoprogression as the initial causes of pleural effusion. The patient had chest wall invasion due to rib metastasis, and the size increased after treatment with atezolizumab. He had a larger rib mass on the left side; thus, pleural effusion of the left lung developed earlier, 11 days after atezolizumab treatment. The right pleural effusion developed after 27 days. In general, irAEs require immunosuppressive therapy, such as steroids. We believe that the causes of both occurrences of pleural effusion were secondary responses to rib mass pseudoprogression.

Kolla et al. reported two cases of recurrent pleural effusion as possible manifestations of pseudoprogression after nivolumab therapy in lung cancer (9). These cases 

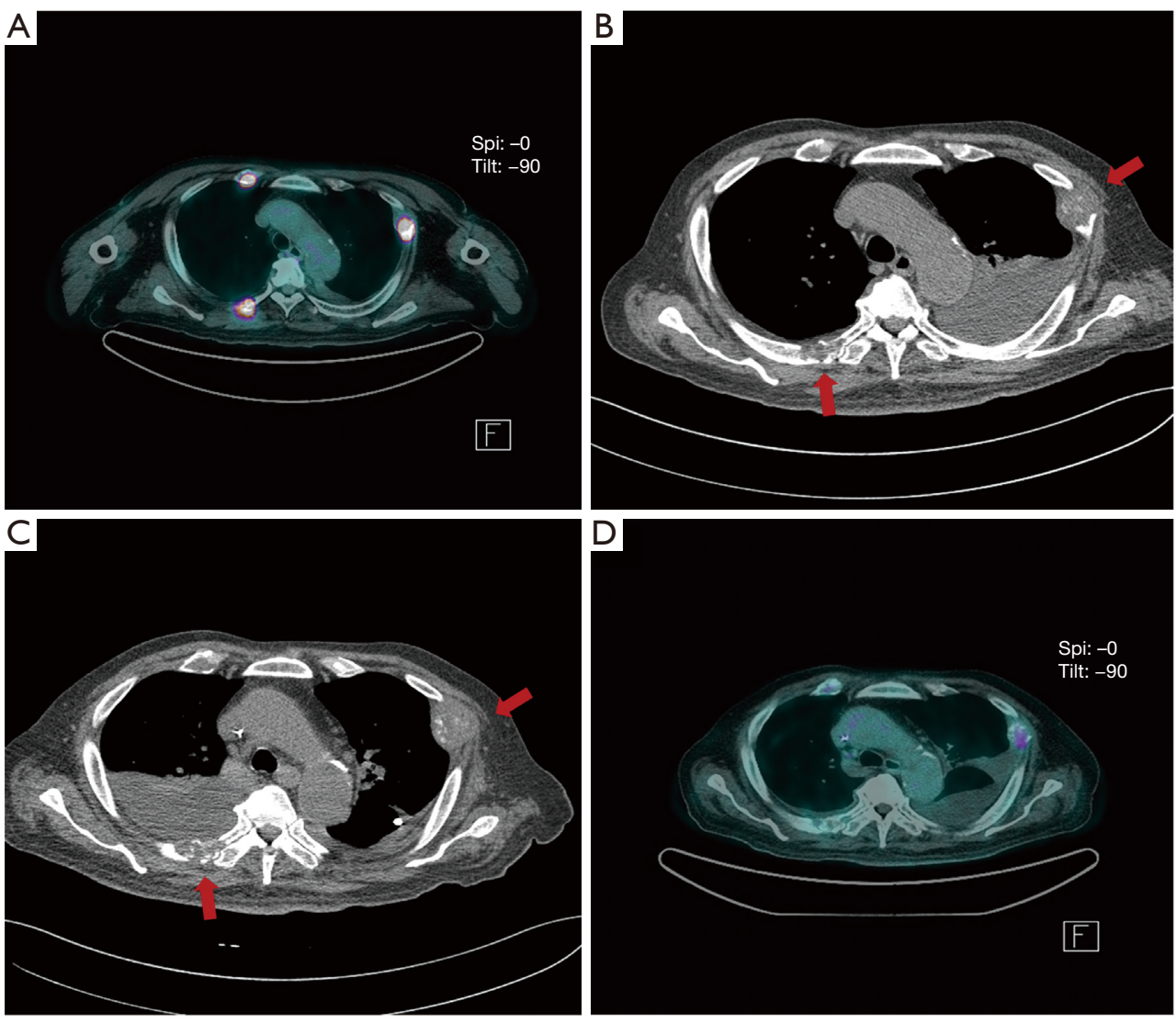

Figure 3 Changes in pleural effusion and rib metastases before and after atezolizumab treatment. (A) PET-CT showed intense FDG uptake in multiple rib metastases before atezolizumab. (B) HRCT showing a moderate amount of pleural effusion on the left lung field and an increase in the size of the left rib metastasis (arrows) 11 days after atezolizumab. (C) HRCT showing a moderate amount of pleural effusion on the right lung field and larger rib metastases (arrows) 27 days after atezolizumab. (D) PET-CT showing that FDG uptake of the rib metastases was significantly lower than that before atezolizumab treatment; left pleural effusion remained mild to moderate 48 days after the first cycle of atezolizumab. PET-CT, positron emission tomography-computed tomography; FDG, fluoro-2-deoxy-D-glucose; HRCT, high-resolution computed tomography.

involved patients with pleural metastasis due to lung cancer; however, after a few days of nivolumab treatment, pleural effusion developed rapidly with an increasing lung mass. Thoracentesis was performed frequently for up to 8 weeks after nivolumab treatment; however, the condition resolved spontaneously with a decrease in the size of the lung mass (9).

Dasanu et al. reported a case of bilateral pleural effusion as an irAE in malignant melanoma, characterized by a moderate amount of bilateral pleural effusion and pericardial effusion, 6 months after completing ipilimumab therapy (10). She was treated with systemic steroids, and the pleural and pericardial effusions resolved two weeks later (10).

Our patient showed spontaneous resolution of pleural effusion by only pleural fluid drainage with PCD without steroids or immunosuppressive drugs at 7 weeks after atezolizumab, similar to the pseudoprogression cases.

Although there have been many studies on pseudoprogression, the exact molecular mechanism has not yet been established. The major mechanism involves the infiltration of immune cells, such as CD4 T cells, CD8 T cells, and macrophages. After ICI therapy, activated immune cells infiltrate the tumor lesion to kill cancer cells, and more inflammatory cells are induced by antigens secreted from dead cancer cells. During this process, vascular tears and hemorrhage can be induced in the tumor tissue, and edema occurs due to inflammatory reactions and hemorrhage. Necrotic byproducts are generated from dead cancer cells, and the unabsorbed portion can accumulate in the 


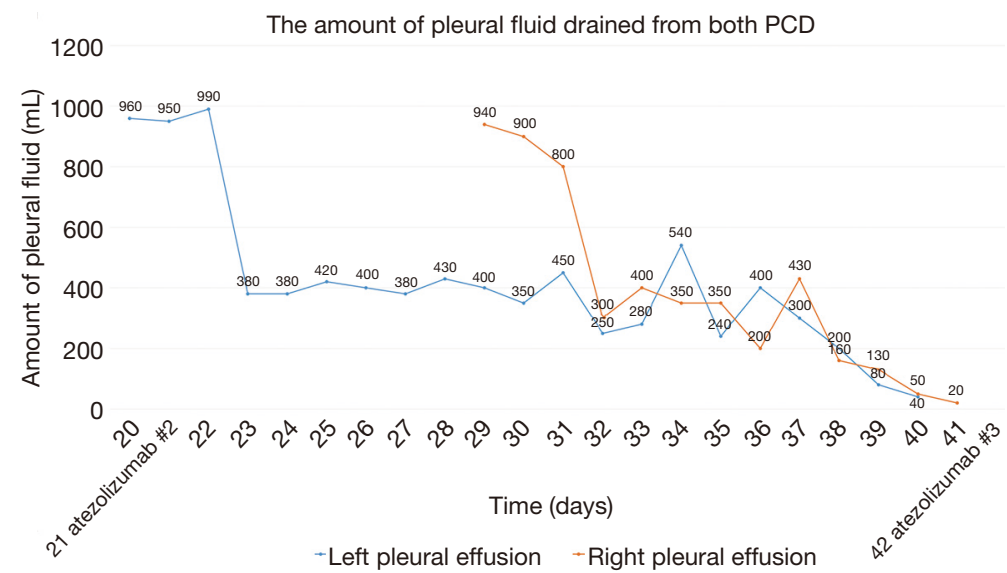

Figure 4 The amount of pleural fluid drained from both PCD. PCD, percutaneous catheter drainage; atezolizumab \#2, the second cycle of atezolizumab; atezolizumab \#3, the third cycle of atezolizumab.

lesion. Inflammatory cell infiltration, hemorrhage, edema, and necrotic byproducts can temporarily enlarge the tumor lesion, which can be observed radiologically as a pseudoprogression (11).

Moreover, the patient presented with LS. UC is the third most common type of cancer in LS-associated tumors, and UC linked to LS is the most common in the upper urinary tract (UUT) (3). The cumulative lifetime risk of developing UC of the UUT is 7.6-22-fold higher than that in the general population (12). LS-related UC is known to develop more commonly in younger and female patients with $M S H 2$ variants $(13,14)$. However, this patient was a relatively elderly man with mutations in $M L H 1$ and $M S H 2$.

ICIs are expected to be used as a central therapeutic regimen for LS-associated cancers. Le et al. found that patients with MMR deficiency showed a significantly higher overall response rate (ORR) $(40 \%)$ than patients with MMR proficiency (11\%) in colon cancer in response to pembrolizumab (2). In this cohort, MMR deficiency was a significant predictor of ORR. In a phase II study that observed the effects of nivolumab plus ipilimumab in patients with MSI-H or MMR-deficient metastatic colorectal cancer, including 56\% with LS-related cancers, Cohen et al. found that the 12-month progression-free survival (PFS) and overall survival (OS) were $76.5 \%$ and $84 \%$, respectively (15). Combined ICI therapy resulted in impressive outcomes in disease control. Only two patients showed pseudoprogression $(3.5 \%)$ in the above study. The first occurred at week 6 in a 68 -year-old man who was MLH1/PMS2-negative, and the other occurred at week 36 in a 47-year-old woman who was MSH2/MSH6-negative. In conclusion, this study showed that the incidence of pseudoprogression in MSI/dMMR may be rare.

There are no clinical studies on the efficacy of ICIs in LS-associated UC, but some case reports have shown them to be effective $(3,16,17)$. This patient was expected to respond to atezolizumab treatment as he had ureteral cancer with LS and as his symptoms did not worsen, ICI therapy was maintained. However, in patients whose prognosis cannot be predicted, if the lesion size rapidly increases after ICI therapy and pleural effusion occurs, this may be a sign of hyperprogression in response to ICI (18), and therefore, change in treatment plan must be considered.

Consequently, further research is needed on the predictive factors that can be expected to show a good response to ICI therapy, and finding patients with such factors is also expected to have a significant effect on treatment and prognosis.

In addition, even if the apparent progression of the disease is likely to be pseudoprogression, it is important to perform accurate response evaluation through close monitoring and, if necessary, re-biopsy.

\section{Acknowledgments}

This paper was supported by the Research Institute of Clinical Medicine of Jeonbuk National University Biomedical Research Institute, Jeonbuk National University Hospital.

Funding: None. 


\section{Footnote}

Reporting Checklist: The authors have completed the CARE reporting checklist. Available at https://apm.amegroups. com/article/view/10.21037/apm-21-1148/rc

Peer Review File: Available at https://apm.amegroups.com/ article/view/10.21037/apm-21-1148/prf

Conflicts of Interest: All authors have completed the ICMJE uniform disclosure form (available at https://apm. amegroups.com/article/view/10.21037/apm-21-1148/coif). The authors have no conflicts of interest to declare.

Ethical Statement: The authors are accountable for all aspects of the work in ensuring that questions related to the accuracy or integrity of any part of the work are appropriately investigated and resolved. All procedures performed in this study were in accordance with the ethical standards of the institutional and/or national research committee(s) and with the Helsinki Declaration (as revised in 2013). Written informed consent was obtained from the patient for publication of this case report and accompanying images. A copy of the written consent is available for review by the editorial office of this journal.

Open Access Statement: This is an Open Access article distributed in accordance with the Creative Commons Attribution-NonCommercial-NoDerivs 4.0 International License (CC BY-NC-ND 4.0), which permits the noncommercial replication and distribution of the article with the strict proviso that no changes or edits are made and the original work is properly cited (including links to both the formal publication through the relevant DOI and the license). See: https://creativecommons.org/licenses/by-nc-nd/4.0/.

\section{References}

1. Lynch HT, Snyder CL, Shaw TG, et al. Milestones of Lynch syndrome: 1895-2015. Nat Rev Cancer 2015;15:181-94.

2. Le DT, Uram JN, Wang H, et al. PD-1 Blockade in Tumors with Mismatch-Repair Deficiency. N Engl J Med 2015;372:2509-20.

3. Lindner AK, Schachtner G, Tulchiner G, et al. Lynch Syndrome: Its Impact on Urothelial Carcinoma. Int J Mol Sci 2021;22:531.

4. Inman BA, Longo TA, Ramalingam S, et al. Atezolizumab:
A PD-L1-Blocking Antibody for Bladder Cancer. Clin Cancer Res 2017;23:1886-90.

5. Seymour L, Bogaerts J, Perrone A, et al. iRECIST: guidelines for response criteria for use in trials testing immunotherapeutics. Lancet Oncol 2017;18:e143-52.

6. Powles T, Durán I, van der Heijden MS, et al. Atezolizumab versus chemotherapy in patients with platinum-treated locally advanced or metastatic urothelial carcinoma (IMvigor211): a multicentre, open-label, phase 3 randomised controlled trial. Lancet 2018;391:748-57.

7. Rosenberg JE, Hoffman-Censits J, Powles T, et al. Atezolizumab in patients with locally advanced and metastatic urothelial carcinoma who have progressed following treatment with platinum-based chemotherapy: a single-arm, multicentre, phase 2 trial. Lancet 2016;387:1909-20.

8. Borcoman E, Nandikolla A, Long G, et al. Patterns of Response and Progression to Immunotherapy. Am Soc Clin Oncol Educ Book 2018;38:169-78.

9. Kolla BC, Patel MR. Recurrent pleural effusions and cardiac tamponade as possible manifestations of pseudoprogression associated with nivolumab therapy- a report of two cases. J Immunother Cancer 2016;4:80.

10. Dasanu CA, Jen T, Skulski R. Late-onset pericardial tamponade, bilateral pleural effusions and recurrent immune monoarthritis induced by ipilimumab use for metastatic melanoma. J Oncol Pharm Pract 2017;23:231-4.

11. Jia W, Gao Q, Han A, et al. The potential mechanism, recognition and clinical significance of tumor pseudoprogression after immunotherapy. Cancer Biol Med 2019;16:655-70.

12. Ito T, Kono K, Eguchi H, et al. Prevalence of Lynch syndrome among patients with upper urinary tract carcinoma in a Japanese hospital-based population. Jpn J Clin Oncol 2020;50:80-8.

13. Therkildsen C, Eriksson P, Höglund M, et al. Molecular subtype classification of urothelial carcinoma in Lynch syndrome. Mol Oncol 2018;12:1286-95.

14. Carlo MI, Ravichandran V, Srinavasan P, et al. Cancer Susceptibility Mutations in Patients With Urothelial Malignancies. J Clin Oncol 2020;38:406-14.

15. Cohen R, Bennouna J, Meurisse A, et al. RECIST and iRECIST criteria for the evaluation of nivolumab plus ipilimumab in patients with microsatellite instability-high/ mismatch repair-deficient metastatic colorectal cancer: the GERCOR NIPICOL phase II study. J Immunother Cancer 2020;8:e01499.

16. Feng Y, Cao Y, Yuan M, et al. Different responses to anti- 
programmed cell death protein 1 (PD-1) immunotherapy in a patient with Lynch syndrome and metachronous urothelial and colon cancer: A case report. Oncol Lett 2019;18:5085-90.

17. Yang Y, Jain RK, Glenn ST, et al. Complete response to anti-PD-L1 antibody in a metastatic bladder cancer

Cite this article as: Yhim HY, Jeon SY, Lee CH, Lee NR. Bilateral pleural effusion associated with atezolizumab in a patient with Lynch syndrome-related urothelial carcinoma: a case report. Ann Palliat Med 2022;11(6):2162-2169. doi: 10.21037/ apm-21-1148 associated with novel MSH4 mutation and microsatellite instability. J Immunother Cancer 2020;8:e000128.

18. Toki MI, Syrigos N, Syrigos K. Hyperprogressive disease: A distinct pattern of progression to immune checkpoint inhibitors. Int J Cancer 2021;149:277-86. 